\title{
A FIXED POINT THEOREM FOR GENERALIZED METRIC SPACES
}

\author{
B. E. RHOADES \\ Department of Mathematics \\ Indiana University \\ Bloomington, Indiana 47405, U.S.A.
}

(Received May 23, 1994)

ABSTRACT. In this paper we prove two fixed point theorems for the generalized metric spaces introduced by Dhage.

In a recent paper, Dhage [1] defined a generalized metric space as follows: Let $D: X \times X \times X \rightarrow \mathbb{R}$ with the following properties:

(i) $D(x, y, z) \geq 0$ for each $x, y, z \in X$, with equality if and only if $x=y=z$,

(ii) $D(x, y, z)=D(y, x, z)=D(x, z, y)=\cdots$ (symmetry)

(iii) $D(x, y, z) \leq D(x, y, a)+D(x, a, z)+D(a, y, z)$, for each $x, y, z \in X$.

2-metric spaces are defined by a function $d: X \times X \times X \rightarrow \mathbb{R}$ with properties (ii) and (iii) above, and (i) replaced by

(i') For each distinct pair $x, y \in X$, there exists a $z \in X$ such that $d(x, y, z) \neq 0$, and $d(x, y, z)=0$ if any two of the triplet $x, y, z$ are equal.

A number of fixed point theorems have been proved for 2-metric spaces. However, Hsiao [2] showed that all such theorems are trivial in the sense that the iterations of $f$ are all colinear. The situation for $D$-metric spaces is quite different. Some specific examples of $D$-metric spaces appear in [1].

The purpose of this paper to prove two general fixed point theorems for $D$-metric spaces.

THEOREM 1. Let $X$ be a complete and bounded $D$-metric space, $f$ a selfmap of $X$ satisfying

$$
\begin{gathered}
D(T x, T y, T z) \leq q \max \{D(x, y, z), D(x, T x, z), D(y, T y, z), \\
D(x, T y, z), D(y, T x, z)\}
\end{gathered}
$$

for all $x, y, z \in X, 0 \leq q<1$. Then $T$ has a unique fixed point $p$ in $X$, and $T$ is continuous at $p$.

PROOF. Let $x_{0} \in X$ and define $x_{n+1}=T x_{n}$. If $x_{n+1}=x_{n}$ for some $n$, then $T$ has a fixed point. Assume that $x_{n+1} \neq x_{n}$ for each $n$. In (1), setting $x=x_{n-1}, y=x_{n}, z=x_{n+p}$, we have

$$
\begin{aligned}
D\left(x_{n}, x_{n+1}, x_{n+p}\right) \leq q & \max \left\{D\left(x_{n-1}, x_{n}, x_{n+p-1}\right), D\left(x_{n-1}, x_{n}, x_{n+p-1}\right)\right. \\
& D\left(x_{n}, x_{n+1}, x_{n+p-1}\right), D\left(x_{n-1}, x_{n+1}, x_{n+p-1}\right) \\
& \left.D\left(x_{n}, x_{n}, x_{n+p-1}\right)\right\}
\end{aligned}
$$

But

$$
\begin{gathered}
D\left(x_{n-1}, x_{n}, x_{n+p-1}\right) \leq q \max \left\{D\left(x_{n-2}, x_{n-1}, x_{n+p-2}\right), D\left(x_{n-2}, x_{n-1}, x_{n+p-2}\right),\right. \\
D\left(x_{n-1}, x_{n}, x_{n+p-2}\right), D\left(x_{n-2}, x_{n}, x_{n+p-2}\right), \\
\left.D\left(x_{n-1}, x_{n-1}, x_{n+p-2}\right)\right\},
\end{gathered}
$$




$$
\begin{gathered}
D\left(x_{n}, x_{n+1}, x_{x+p-1} \leq q \max \left\{D\left(x_{n-1}, x_{n}, x_{n+p-2}\right), D\left(x_{n-1}, x_{n}, x_{n+p-2}\right),\right.\right. \\
D\left(x_{n}, x_{n+1}, x_{n+p-2}\right), D\left(x_{n-1}, x_{n+1}, x_{n+p-2}\right), \\
\left.D\left(x_{n}, x_{n}, x_{n+p-2}\right)\right\}, \\
D\left(x_{n-1}, x_{n+1}, x_{n+p-1}\right) \leq q \max \left\{D\left(x_{n-2}, x_{n}, x_{n+p-2}\right), D\left(x_{n-2}, x_{n-1}, x_{n+p-2}\right),\right. \\
D\left(x_{n}, x_{n+1}, x_{n+p-2}\right), D\left(x_{n-2}, x_{n+1}, x_{n+p-2}\right), \\
\left.D\left(x_{n}, x_{n-1}, x_{n+p-1}\right)\right\},
\end{gathered}
$$

and

$$
D\left(x_{n}, x_{n}, x_{n+p-1}\right) \leq q \max \left\{D\left(x_{n-1}, x_{n-1}, x_{n+p-2}\right), D\left(x_{n-1}, x_{n}, x_{n+p-2}\right)\right\} .
$$

Substituting (3) - (6) into (2) gives

$$
D\left(x_{n}, x_{n+1}, x_{n+p}\right) \leq q^{2} \max _{a, b, c} D\left(x_{a}, x_{b}, x_{c}\right),
$$

where $n-2 \leq a \leq n, n-1 \leq b \leq n+1$, and $c=n+p-2$. Continuing this process it follows that

$$
D\left(x_{n}, x_{n+1}, x_{n+p-1}\right) \leq q^{n} \max _{a, b, c} D\left(x_{a}, x_{b}, x_{c}\right)
$$

where now $0 \leq a \leq n, 1 \leq b \leq n+1$, and $c=p$. Let $M:=\sup _{x, y, z \in X} D(x, y, z)$. Then, it follows from (7) that

$$
D\left(x_{n}, x_{n+1}, x_{n+p}\right) \leq q^{n} M
$$

Using (iii) and (8),

$$
\begin{aligned}
D\left(x_{n}, x_{n+p}, x_{n+p+t}\right) \leq & D\left(x_{n}, x_{n+p}, x_{n+1}\right)+D\left(x_{n}, x_{n+1}, x_{n+p+t}\right)+D\left(x_{n+1}, x_{n+p}, x_{n+p+t}\right) \\
\leq & 2 M q^{n}+D\left(x_{n+1}, x_{n+p}, x_{n+p+t}\right) \\
\leq & 2 M q^{n}+D\left(x_{n+1}, x_{n+p}, x_{n+2}\right)+D\left(x_{n+1}, x_{n+2}, x_{n+p+t}\right) \\
& \quad+D\left(x_{n+2}, x_{n+p}, x_{n+p+t}\right) \\
\leq & 2 M\left(q^{n}+q^{n+1}\right)+D\left(x_{n+2}, x_{n+p}, x_{n+p+1}\right) \leq \cdots \\
\leq & 2 M\left(q^{n}+q^{n+1}+\cdots+q^{n+p-1}\right)+D\left(x_{n+p-1}, x_{n+p}, x_{n+p+t}\right) \\
\leq & 2 M \sum_{k=n}^{n+p} q^{k} \leq \frac{2 M q^{n}}{1-q} \rightarrow 0 \text { as } n \rightarrow \infty
\end{aligned}
$$

Therefore $\left\{x_{n}\right\}$ is $D$-Cauchy. Since $X$ is complete, $\left\{x_{n}\right\}$ converges. Call the limit $p$.

From (1),

$$
D\left(x_{n}, x_{n+1}, T p\right) \leq q \max \left\{D\left(x_{n-1}, x_{n}, p\right), D\left(x_{n}, x_{n+1}, p\right), D\left(x_{n-1}, x_{n+1}, p\right), D\left(x_{n}, x_{n}, p\right)\right\} .
$$

Taking the limit as $n \rightarrow \infty$, and using the fact that $D$ is continuous, yields $D(p, p, T p) \leq 0$, which implies that $p=T p$.

To prove uniqueness, assume that $w \neq p$ is also a fixed point of $T$. From (1),

$$
\begin{aligned}
& D(p, w, p)=D(T p, T w, T p) \\
& \quad \leq q \max \{D(p, w, p), D(p, T p, p), D(w, T w, p), D(p, T w, p), D(w, T p, p)\} \\
& \quad=q \max \{D(p, w, p), D(w, w, p)\}=q D(w, w, p) .
\end{aligned}
$$

But

$$
\begin{aligned}
& D(w, w, p)=D(w, p, w)=D(T w, T p, T w) \\
& \quad \leq q \max \{D(w, p, w), D(w, T w, w), D(p, T p, w), D(w, T p, w), D(p, T w, w)\} \\
& \quad=q \max \{D(w, p, w), D(p, p, w)\}=q D(p, p, w)
\end{aligned}
$$


Combining (9) and (10) yiclds $D(p, w, p) \leq q^{2} D(p, w, p)$, a contradiction. Therefore $p=u$.

To show that $T$ is continuous at $p$, let $\left\{y_{n}\right\} \subseteq X$ with $\lim y_{n}=p$. Then, substituting in (1), with $x=z=p, y=y_{n}$, we obtain

$$
\begin{gathered}
D\left(T p, T y_{n}, T p\right) \leq q \max \left\{D\left(p, y_{n}, p\right), D(p, T p, p), D\left(y_{n}, T y_{n}, p\right),\right. \\
\left.D\left(p, T y_{n}, p\right), D\left(y_{n}, T p, p\right)\right\}
\end{gathered}
$$

Taking the limsup of (11), we obtain

$$
\lim \sup D\left(p, T y_{n}, p\right) \leq q \max \left\{0,0, \lim \sup D\left(p, T y_{n}, p\right), 0\right\},
$$

which implies that $\lim T y_{n}=p=T p$, and $T$ is continuous at $p$.

COROLLARY 1. Let $X$ be a complete and bounded $D$-metric space, $m$ a positive integer, $T$ a selfmap of $X$ satisfying

$$
\begin{gathered}
D\left(T^{m} x, T^{m} y, T^{m} z\right) \leq q \max \left\{D(x, y, z), D\left(x, T^{m} x, z\right), D\left(y, T^{m} y, z\right),\right. \\
\left.D\left(x, T^{m} y, z\right), D\left(y, T^{m} x, z\right)\right\}
\end{gathered}
$$

for all $x, y, z \in X, 0 \leq q<1$. Then $T$ has a unique fixed point $p$ in $X$, and $T^{m}$ is continuous at $p$.

PROOF. From Theorem $1, T^{m}$ has a unique fixed point $p$, and $T^{m}$ is continuous at $p$. But $T p=T\left(T^{m} p\right)=T^{m}(T p)$, and $T p$ is also a fixed point of $T^{m}$. Since the fixed point is unique, $p=T p$.

THEOREM 2. Let $X$ be a compact $D$-metric space, $f$ a continuous selfmap of $X$ satisfying

$$
\begin{gathered}
D(T x, T y, T z)<\max \{D(x, y, z), D(x, T x, z), D(y, T y, z), \\
D(x, T y, z), D(y, T x, z)\}
\end{gathered}
$$

for all $x, y, z \in X$. Then $T$ has a unique fixed point $p$ in $X$.

PROOF. Since $X$ is compact, both sides of (12) are bounded.

Case I. Suppose that the right-hand-side of (12) is positive for all $x, y, z$ in $X$. Define

$$
f(x, y, z):=\frac{D(T x, T y, T z)}{\max \{D(x, y, z), D(x, T x, z), D(y, T y, z), D(x, T y, z), D(y, T x, z)\}} .
$$

Since $T$ and $D$ are continuous, so is $f$. The compactness of $X$ implies that $f$ assumes its maximum at some point $(u, v, w)$ in $X$. Call the value $c$. From (12), it follows that $0<c<1$. Thus $T$ now satisfies (1) with $q=c$. By Theorem $1, T$ has a unique fixed point $p$.

Case II. Suppose there exists a point $(x, y, z)$ such that the right-hand-side of (12) is zero. Then, in particular, $x=T x=z$, and $x$ is a fixed point of $T$. Suppose that $w$ is also a fixed point of $T$. Then, using the same argument as in Theorem 1 , it follows that $x=w$, and the fixed point is unique.

COROLLARY 2. Let $X$ be a compact $D$-metric space, $m$ a positive integer, $T$ a continuous selfmap of $X$ satisfying

$$
\begin{gathered}
D\left(T^{m} x, T^{m} y, T^{m} z\right)<\max \left\{D(x, y, z), D\left(x, T^{m} x, z\right), D\left(y, T^{m} y, z\right)\right. \\
\left.D\left(x, T^{m} y, z\right), D\left(y, T^{m} x, z\right)\right\}
\end{gathered}
$$


for all $x, y, z \in X$. Then $T$ has a unique fixed point $p$ in $X$.

The proof of Corollary 2 parallels that of Corollary 1.

Theorem 2.1 and 2.2 of Dhage [1] are special cases of Theorems 1 and 2 of this paper.

There are two limitations involving fixed point theorems on $D$-metric spaces. The first is that the proof of the existence of a fixed point appears to require that $X$ be bounded. The sccond is that there is apparently no reasonable contractive definition for a pair of maps on a $D$-metric space.

\section{REFERENCES}

1. DHAGE, B. C. Generalized metric spaces and mappings with fixed point, Bull. CalcuttaMath.Soc. 84 (1992), 329-336.

2. HSIAO, C.-R. A property of contractive type mappings in 2-metric spaces, Jnanabha 16 (1986), 223-239. 


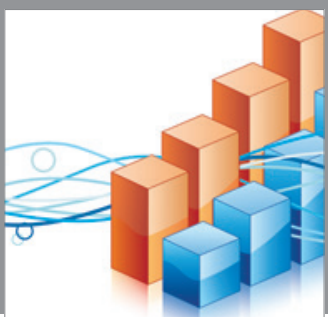

Advances in

Operations Research

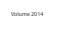

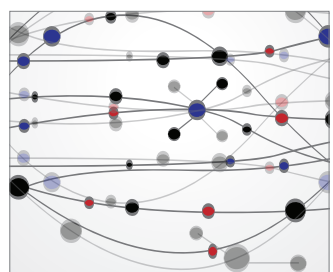

\section{The Scientific} World Journal
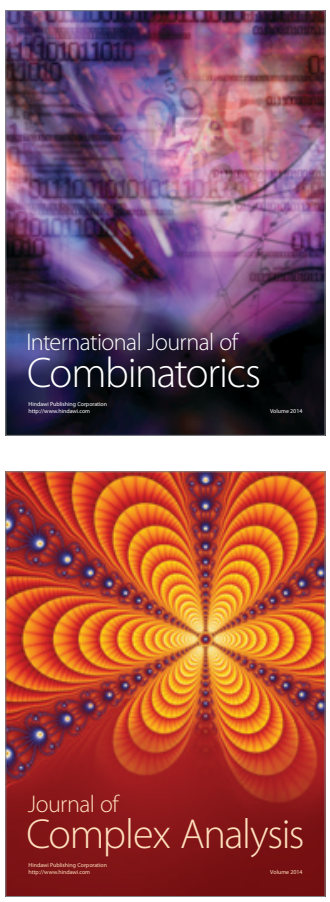

International Journal of

Mathematics and

Mathematical

Sciences
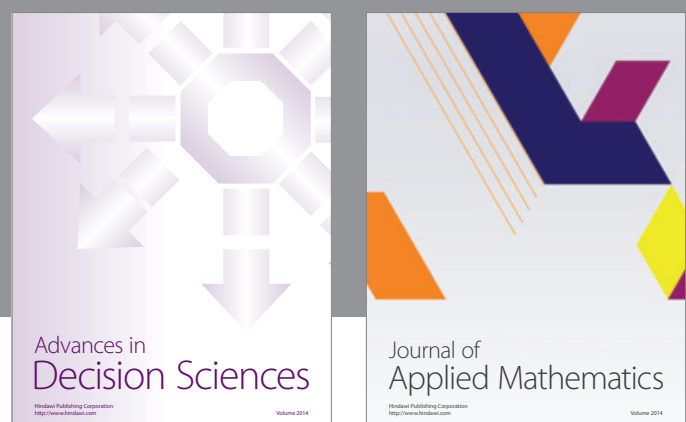

Journal of

Applied Mathematics
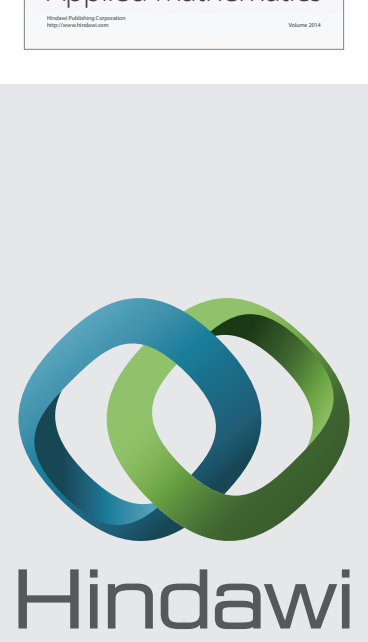

Submit your manuscripts at http://www.hindawi.com
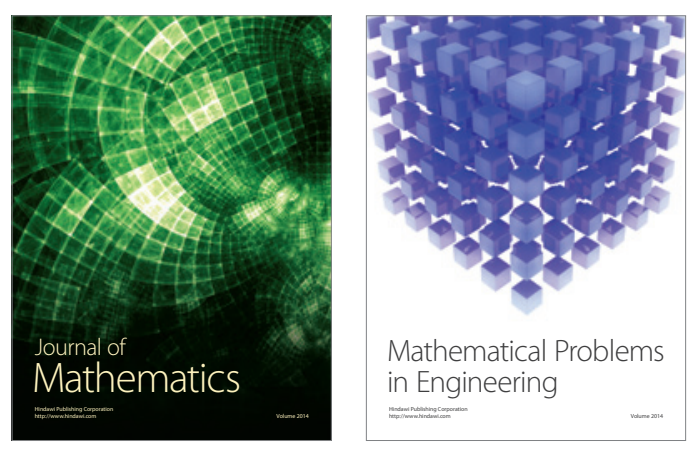

Mathematical Problems in Engineering
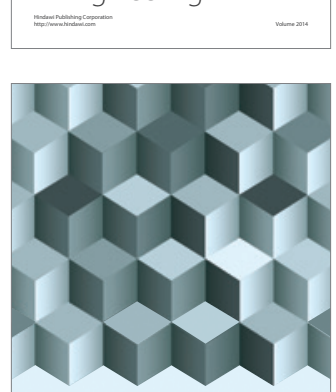

Journal of

Function Spaces
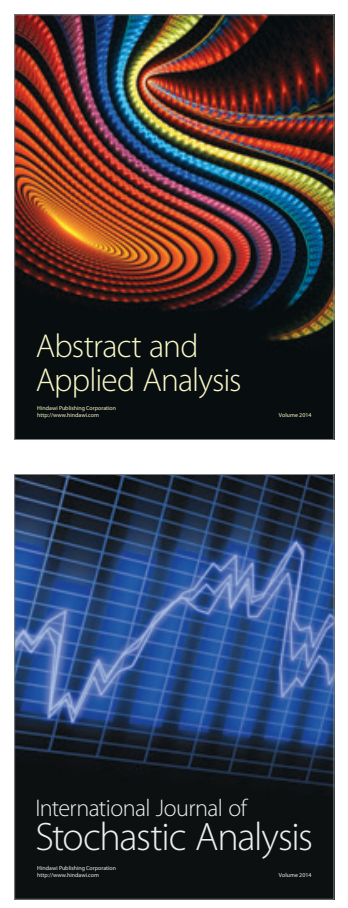

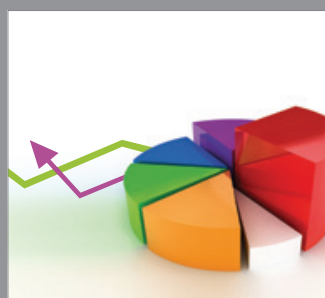

ournal of

Probability and Statistics

Promensencen
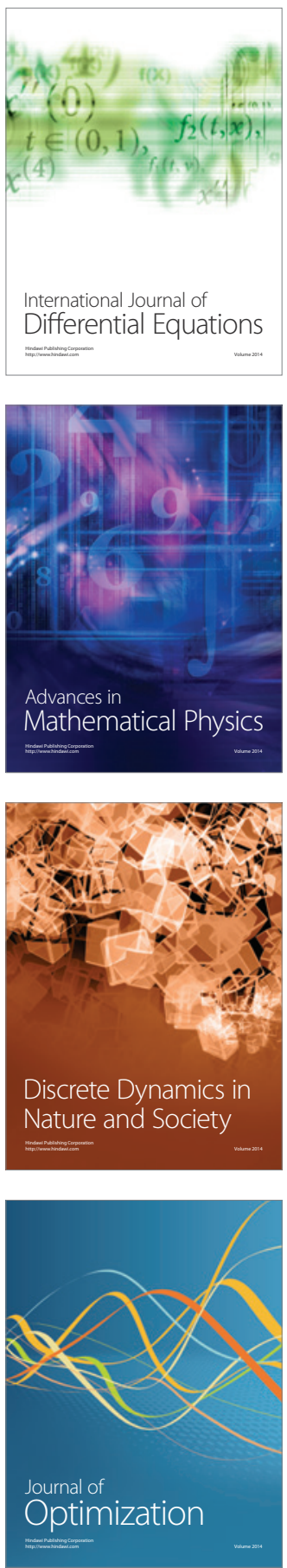